\title{
Emisiones Máseres en Regiones de Formación de Estrellas de Gran Masa: ¿Trazadores de Discos o de "Jets"?
}

\author{
Jesús Umanzor ${ }^{11}$ \\ Jesuma_78@yahoo.com \\ Mercedes Gómez²2 \\ mercedes@oac.uncor.edu \\ ${ }^{1}$ Observatorio Astronómico Centroamericano de Suyapa. \\ Universidad Nacional Autónoma de Honduras \\ 2 Observatorio Astronómico de la Universidad Nacional de Córdoba
}

\section{Resumen}

En un trabajo previo presentamos un estudio, con el cual pretendíamos determinar si los máseres de metanol, hidroxilo y agua se alínean en un número significativo de regiones más frecuentemente con las estructuras de discos o "jets" que acompañan el proceso de formación de las estrellas de masa alta de nuestra Galaxia. Los resultados de tal estudio indican que las emisiones de máseres que circundan las protoestrellas de masa alta tienden a formar grupos que en un porcentaje muy elevado muestran distribuciones lineales 0 elongadas. La linealidad de las distribuciones de los máseres es tan frecuente que es consistente con la idea que los máseres están vinculados o se producen en los discos o "jets". Sin embargo, encontramos que no existe una marcada preferencia de los máseres a alinearse con alguno de estos tipos de estructuras. En esta contribución se presentan los resultados de 8 nuevas regiones, encontramos que los resultados obtenidos anteriormente se mantienen, es decir que los máseres se alinean con discos y "jets" en proporciones similares.

Palabras clave: estrellas de gran masa, formación estelar, máseres, discos, "jets/outflows"

\begin{abstract}
A previous study this one, with which it was intended to determine whether masers of methanol, hydroxyl and water align in a significant number of the regions most frequently with disk structures or "jets" that accompany the process of formation of high-mass stars. The results of this study indicate that emissions of masers around the proto-stars of high mass tend to form groups at a very high percentage distributions are linear or elongated. The linearity of the distributions of the masers is so frequent that it is consistent with the idea that the masers are linked or occur in the discs or jets. However we did not find a significant preference of the masers of methanol, hydroxyl and water to align with disks and jets. This contribution presents the results of 8 new regions, we find that past performance is maintained, meaning that the masers are aligned with disks and jets in similar proportions.
\end{abstract}

Key words: stars of high mass, formation stellar, masers, disks, jets/outflows

\footnotetext{
${ }^{1}$ Observatorio Astronómico Centroamericano de Suyapa.

Universidad Nacional Autónoma de Honduras

${ }^{2}$ Observatorio Astronómico de la Universidad Nacional de Córdoba
} 


\section{Introducción}

La formación de las estrellas de gran masa está asociada a fenómenos como máseres, "jets" y discos. En los últimos años se busca determinar el rol que juegan estos en el proceso de formación de tales estrellas. En este trabajo se presentan los resultados de un estudio que intenta establecer si existe alineación preferencial de las emisiones máseres de metanol $\left(\mathrm{CH}_{3} \mathrm{OH}\right)$, hidroxilo $(\mathrm{OH})$ y agua $\left(\mathrm{H}_{2} \mathrm{O}\right)$ con respecto a las estructuras de flujos bipolares moleculares ("jets": en el óptico y "outflow": en longitudes de onda de radio) y discos circunestelares. En la Figura 1 se muestra una representación esquemática de los escenarios actuales que vinculan las emisiones de máseres con los "jets" (panel izquierdo) y/o con discos (panel derecho).
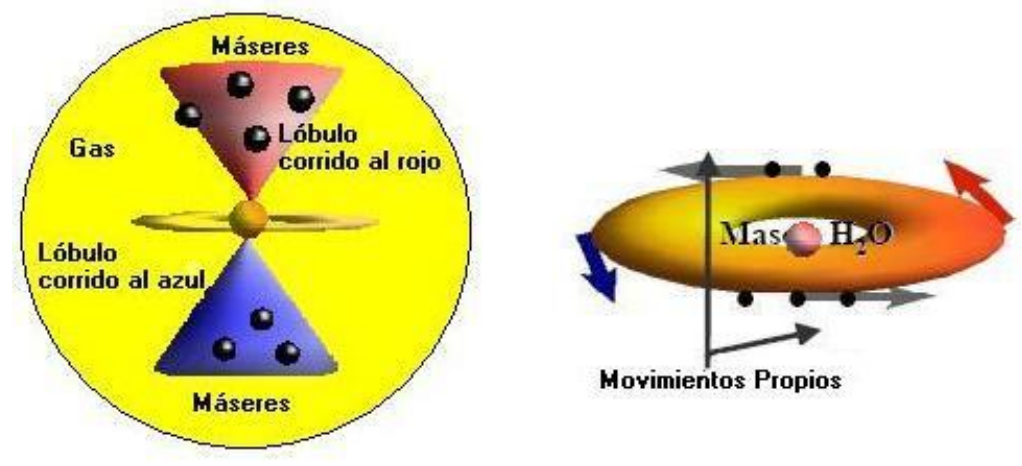

Figura 1: Representación esquemática de máseres asociados con "jets" y discos. Panel Izquierdo: Máseres asociados con "jets". Panel Derecho: Máseres asociados con "discos". Esta figura fue adaptada del trabajo de Goddi et al. (2005)

\section{Metodología}

En el presente trabajo se busca determinar correlaciones de alineamiento entre distribuciones de máseres de metanol, hidroxilo y agua con respecto a las estructuras de "jets" y discos que acompañan a la formación de las estrellas de gran masa. Para alcanzar tal propósito resulta necesario hacer una búsqueda y recopilación bibliográfica de las posiciones de los máseres en cada región, en particular de aquellos que yacen más cercanos al centro de las mismas. Así mismo, también de la bibliografía disponible para cada región se obtienen los ángulos de posición de los "jets" y discos asociados a cada una de las proto-estrellas de gran masa analizadas. Inicialmente, y a los fines de realizar un análisis preliminar de los máseres de cada región, se consultaron diversos catálogos disponibles en la base de datos SIMBAD ("SIMBAD Astronomical Database"). Posteriormente y con el fin de obtener las posiciones de los máseres relativamente más cercanos al centro de cada una de las regiones y disponer de los relevamientos más recientes, se consultan todas las publicaciones individuales de cada región utilizando la base de datos ADS ("The Astronomical Database System"). En general, estas publicaciones además de proporcionar las posiciones de los distintos tipos de máseres en cada región también proporcionan los ángulos de posición de los discos y de los "jets" en cada caso. Para facilitar la construcción de los gráficos las posiciones de los máseres en la base de datos son relativas ("offsets") al centro de la región. En algunos casos en la literatura sólo se publican las coordenadas absolutas de las posiciones de los máseres. Para homogenizar los datos es necesario transformar las mismas a posiciones relativas ("offset"). Esto se realiza haciendo uso de las siguientes relaciones:

y son las coordenadas centrales de cada región y y las coordenadas individuales de cada máser. Finalmente e son los desplazamientos ("offsets") que por conveniencia expresamos en segundos de arco. 
Para facilitar la búsqueda de correlaciones entre las direcciones de las estructuras de discos y de "jets" y la distribución de los distintos tipos de máseres de cada región se construye un gráfico donde se muestran las posiciones "offsets" de los máseres en relación a los ángulos de posición de los discos y/o "jets". Este procedimiento permite una inspección visual de cada región y pone en evidencia los alineamientos entre los máseres y los dos tipos de estructuras. Finalmente se realiza un análisis global de la muestra para determinar si los máseres se alinean con los discos o con los "jets/outflows".

\section{Resultados}

En esta sección se presenta un análisis de las 8 regiones (ver Tabla 1) de formación de estrellas de gran masa seleccionadas. En las mismas se han detectado directa o indirectamente estructuras de discos y "jets" asociadas con estrellas de gran masa, además de la presencia de máseres. El objetivo principal es tratar de determinar si los máseres tienden a alinearse preferentemente con alguno de los dos tipos de estructuras mencionados.

En la figura 2 y 3 se presentan los gráficos con las posiciones "offsets" de los máseres (metanol, hidroxilo y agua) en las regiones seleccionadas. También se indican los ángulos de posición de los "jets". En estas figuras los triángulos azules indican las posiciones "offsets" de los máseres de agua; los cuadrados negros los máseres de metanol y los pentágonos rojos las posiciones de los máseres de hidroxilo. Las líneas de color verde indican la dirección de los "jets". A continuación se describen los alineamientos entre las distintas estructuras para cada región.

Tabla 1: Regiones de Formación de Estrellas de Masa Alta Seleccionadas

\begin{tabular}{|l|c|c|}
\hline Región & A. R. (2000) & Dec. (2000) \\
\hline IRAS00338+6312 & 003647.5 & 632902 \\
\hline G139.909+0.197 & 030723.7 & 583050 \\
\hline IRAS06053-0622 & 060748.0 & -062257 \\
\hline IRAS06055+2039 & 060835.5 & 203859 \\
\hline IRAS06056+2131 & 060841.2 & 213104 \\
\hline G192.584-0.041 & 061254.0 & 175923 \\
\hline G70.293+1.6 & 200145.7 & 333243 \\
\hline S138 & 223246.0 & 582822 \\
\hline
\end{tabular}

IRAS 00338+6312: En el panel superior izquierdo de la Figura 2 se observan las posiciones "offsets" de los máseres de metanol, hidroxilo y agua de esta región. Los máseres de metanol siguen la dirección del "jet" de la región. Por otro lado el número de los máseres de hidroxilo y agua es muy bajo como para tratar de buscar alineamientos con el "jet".

G139.909+0.197: En el panel superior derecho de la Figura 2 mostramos las posiciones de los máseres de agua detectados en esta región. Estos máseres se distribuyen linealmente en la dirección Sureste-Noroeste y yacen aproximadamente perpendiculares al "outflow". En esta región no se ha detectado máseres de hidroxilo y metanol. 

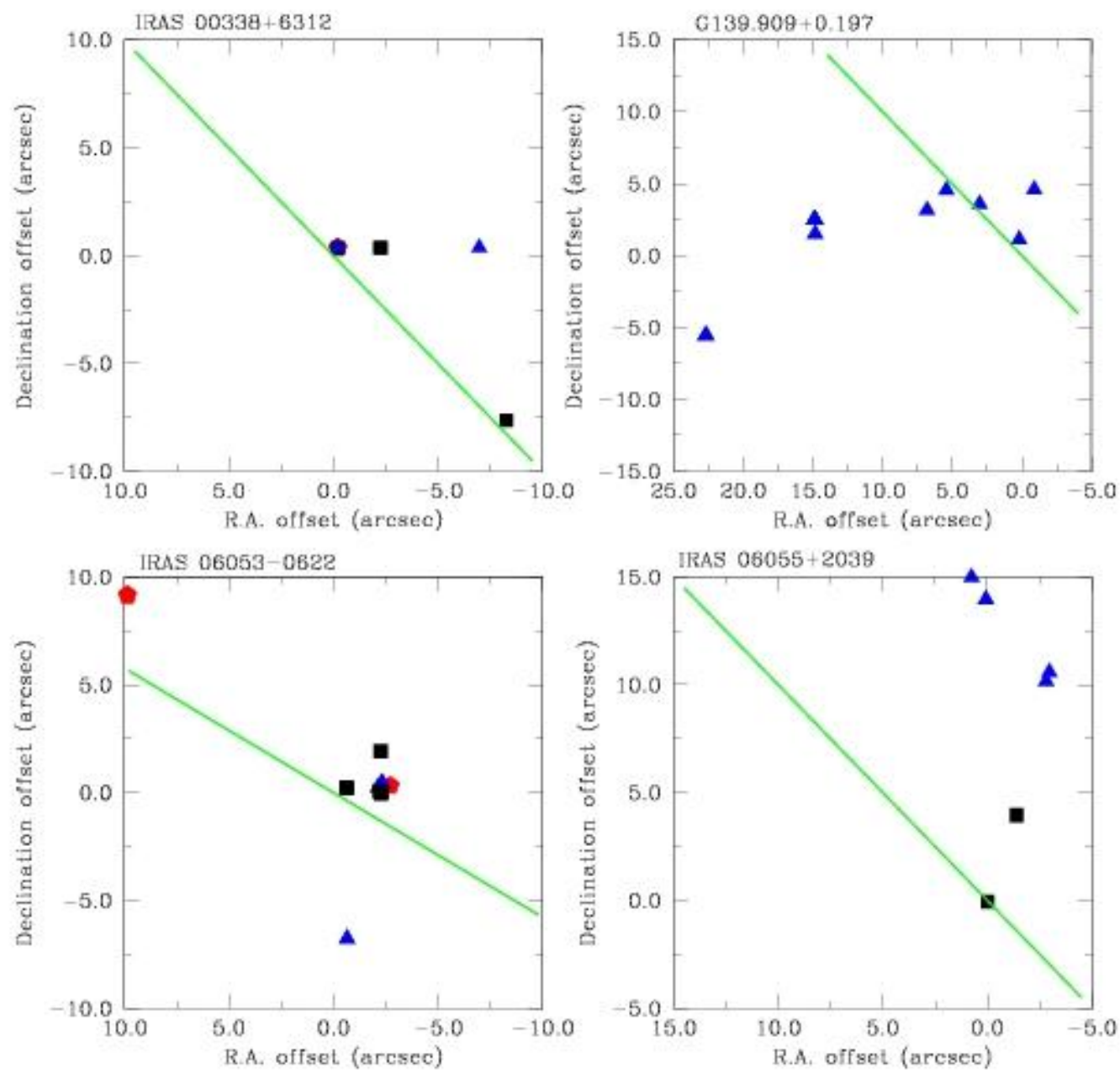

Figura 2: Posiciones "offsets" de los máseres y dirección de los discos y "jets". Los máseres de metanol son indicados por cuadrados negros, los de hidroxilo por pentágonos rojos y los de agua por triángulos azules. La línea de color verde indica la dirección del "jet/outflow". Panel Superior Izquierdo: IRAS 00338+6312 Panel Superior Derecho: G139.909+0.197 Panel Inferior Izquierdo: IRAS 06053-0622 Panel Inferior Derecho: IRAS 06055+2039

IRAS 06053-0622: En el panel inferior izquierdo de la Figura 2 se aprecia la ubicación de los máseres de metanol, hidroxilo y agua de esta región. Los máseres de metanol están cercanamente perpendiculares a la dirección del "jet". En cambio los máseres de hidroxilo y agua son muy pocos para determinar alineamientos.

IRAS06055+2039: En el panel inferior derecho de la Figura 2 se muestran los máseres de metanol y agua de esta región. Al Noroeste de la región se encuentra una distribución lineal de máseres de agua que son paralelos a la dirección del "jet" sin embargo, se encuentran más de 5" alejados del mismo, por lo tanto es muy poco probable que estos máseres estén vinculados con el "jet" de la región. En cambio el número de los máseres de metanol es muy bajo como para tratar de establecer alineamientos.

IRAS06056+2131: En el panel superior izquierdo de la Figura 3 se observan los máseres de metanol y agua de la región. Los máseres de metanol siguen la dirección del "jet". Al suroeste yace el único máser de agua de la región. En la misma no se han detectado máseres de hidroxilo.

G192.584-0.041: En el panel superior derecho de la Figura 3 se muestran la distribución de los tres distintos tipos de máseres de la región. En general los máseres de metanol están distribuidos linealmente en una dirección 
cercanamente perpendicular a la dirección del ángulo de posición del "jet". Sugerimos que los mismos estarían siguiendo la dirección de un disco que aun no se ha detectado observacionalmente. Los máseres de agua están distribuidos linealmente sin embargo, no están alineados con el "jet" ni perpendicularmente al mismo. Al Suroeste de la región yace el único máser de metanol.

G70.293+1.6: En el panel inferior izquierdo de la Figura 3 se aprecian los máseres de metanol, hidroxilo y agua de esta región. Los máseres de agua yacen en la misma dirección del "jet". Por otro lado los máseres de hidroxilo están distribuidos en dos grupos el primero al Suroeste de la región siguen la dirección del "jet". El segundo grupo de máseres de hidroxilo se encuentra al Sureste de la región, cerca al único máser de metanol y no están alineados con el "jet" ni perpendicular al mismo.

S138: En el panel inferior derecho de la Figura 3 se muestra el único máser de agua y la dirección del "jets" detectado en esta región. Como el número de máseres es muy bajo en esta región no se pueden determinar alineamientos.
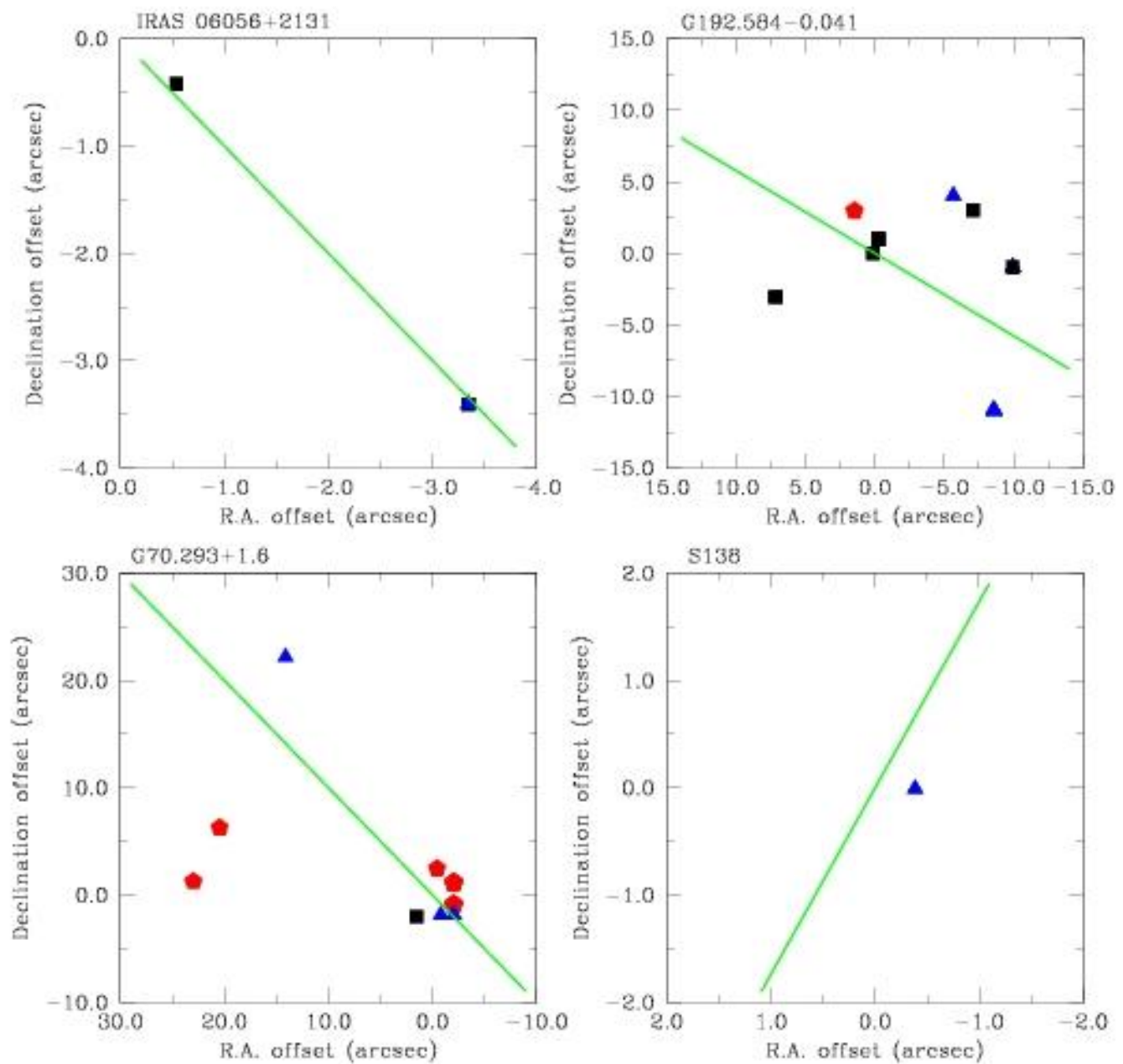

Figura 3: Posiciones "offsets" de los máseres y dirección de los discos y "jets". Los máseres de metanol son indicados por cuadrados negros, los de hidroxilo por pentágonos rojos y los de agua por triángulos azules. La línea de color verde indica la dirección de "jet/outflow". Panel Superior Izquierdo: IRAS 06056+2131 Panel Superior Derecho: G192.584-0.041 Panel Inferior Izquierdo: G70.293+1.6 Panel Inferior Derecho: S138. 
La Tabla 2 resume los resultados encontrados en esta sección. Las columnas 2, 3 y 4 indican la morfología de la distribución de los máseres de metanol, hidroxilo y agua de cada región. Encontramos distribuciones lineales (I), elongadas (e) y complejas (c). Hacemos notar que en porcentaje muy elevado (7 de 8 regiones) poseen distribuciones de máseres lineales o elongadas. Las columnas 5 y 6 detallan el (los) tipo(s) de máser(es) que se alinean con el "jet" o disco de la región en cuestión. Además hacemos notar que en las regiones de la muestra seleccionada no se han detectado directamente discos, solamente "jets".

La Tabla 3 muestra los resultados de los alineamientos de los máseres, discos y "jets". Resulta evidente observar que los máseres de metanol, hidroxilo y agua se alinean con estructuras de discos y de "jets" en proporciones similares. No existe una preferencia marcada de alineamiento de los máseres ni con los discos ni con los "jets". En general encontramos que los máseres pueden trazar tanto discos como "jets".

Umanzor \& Gómez (2008) encontraron en una muestra de 29 regiones de formación de estrellas de masa alta que los máseres metanol, hidroxilo y agua se alinean con las estructuras de discos y "jets" en proporciones similares. Al sumar a esta muestra los resultados de estas 8 nuevas regiones encontramos que esta tendencia se mantiene. Es decir los máseres pueden trazar discos y "jets" en proporciones similares y no hay una marcada preferencia de alineamiento con alguna de estas estructuras.

Tabla 2: Morfología de distribución de máseres y su alineamiento con discos y/o "jets"

\begin{tabular}{|l|c|c|c|c|c|l|}
\hline Región & Metanol & Hidroxilo & Agua & Jet & Disco & Referencia \\
\hline IRAS00338+6312 & I & $*$ & I & m & & 5 \\
\hline G139.909+0.197 & & & e & & a & 3 \\
\hline IRAS06053-0622 & e & I & I & & m & 5 \\
\hline IRAS06055+2039 & & I & I & & & 4,5 \\
\hline IRAS06056+2131 & I & & & m & & 5 \\
\hline G192.584-0.041 & l & $*$ & I & & m & 3 \\
\hline G70.293+1.6 & $*$ & e & I & h; a & & 3 \\
\hline S138 & & & $*$ & & & 3 \\
\hline
\end{tabular}

Tabla 3: Alineamientos entre máseres discos y "jets"

\begin{tabular}{|c|c|c|c|}
\hline Tipo de Máser & Metanol & Hidroxilo & Agua \\
\hline $\mathrm{N}^{\circ}$ de alineamientos con "jets" & 2 & 1 & 1 \\
\hline $\mathrm{N}^{\circ}$ de alineamientos con discos & 2 & 0 & 1 \\
\hline
\end{tabular}

\section{Discusión}

Durante mucho tiempo el estudio observacional de las regiones circundantes a los objetos estelares jóvenes de gran masa se ha visto seriamente limitado ya que estos se encuentran profundamente embebidos o incrustados en nubes de gas denso y polvo. Típicamente las nubes progenitoras poseen altos grados de extinción óptica $\left(A_{v}\right\rangle$ 50-100 mag) y en consecuencia los objetos estelares jóvenes en proceso de formación se vuelven invisibles en el óptico y, por lo tanto son más fáciles de detectar cuando mayor sea la longitud de onda de la radiación estudiada ya que el medio ambiente es transparente. 
Otro de los factores que ha dificultado el estudio de los objetos estelares jóvenes de gran masa es que, en general, estos yacen a grandes distancias (típicamente de algunos Kpc), por lo cual para poderlos observar se requieren instrumentos de alta sensibilidad y resolución angular. Por ejemplo una resolución angular de 0.1 " para objetos a distancias alrededor de $0.5 \mathrm{Kpc}$, corresponde a distancias lineales de alrededor de 0.8 pc $016500 \mathrm{UA}$.

Las emisiones de máseres de especies moleculares tales como $\mathrm{CH}_{3} \mathrm{OH}, \mathrm{OH}$ y $\mathrm{H}_{2} \mathrm{O}$ se producen en zonas de gas de densidad alta $\left(\sim 10^{7}-10^{9} \mathrm{~cm}^{-3}\right)$ y relativamente calientes $\mathrm{T} \sim 100 \mathrm{~K}$. Es decir, que la presencia de máseres en una región de formación de estrellas indica la existencia de condiciones específicas para su excitación. Las transiciones de los máseres ocurren en longitudes de onda centimétricas, en tales longitudes de onda el medio ambiente que circunda a los objetos estelares jóvenes de gran masa en formación es transparente. Además las emisiones de máseres son muy intensas (densidades de flujo de varios Jy) en relación de otras fuentes astronómicas en las mismas longitudes de onda. Gracias a estos factores las emisiones de máseres permiten superar los obstáculos mencionados, resultando posible su detección en regiones de formación de estrellas de gran masa que se encuentran relativamente lejanas y muy oscurecidas por el gas y el polvo de la nube progenitora.

Frecuentemente es posible determinar la posición de los máseres de una región dada con una precisión de submili-segundo de arco. Para fijar ideas para una distancia de $2 \mathrm{Kpc}, 1$ mili-segundo de arco corresponde a una resolución lineal de $0.3 \mathrm{UA}$. Las observaciones de emisiones de máseres constituyen una herramienta muy eficaz para estudiar con gran resolución angular el gas molecular cercano a las protoestrellas de gran masa.

Las condiciones físicas de densidad alta $\sim 10^{7}-10^{9} \mathrm{~cm}^{-3}$ y temperatura de algunos $\mathrm{T} \sim 100 \mathrm{~K}$ necesarias para la producción de máseres son, además, las que caracterizan la parte interna de los discos circunestelares alrededor de los objetos jóvenes de gran masa y a su vez el gas chocado (objetos $\mathrm{HH}$ ), "jets" y/o "outflows", asociados con los vientos estelares de los objetos en formación. Por lo tanto los máseres pueden, en principio, existir en ambos tipos de estructuras. Los mismos podrían entonces utilizarse como trazadores de discos y/o "jets".

En síntesis y dado que los máseres requieren para su excitación de condiciones físicas específicas similares a las que posee el gas molecular cercano a las protoestrellas de gran masa en formación, estos se han convertido en una herramienta clave para su estudio. La observación de emisiones máseres es una técnica muy efectiva no sólo para seleccionar nuevos candidatos a estrellas de gran masa, sino también para trazar las estructuras de discos y/o "jets" que acompañan el proceso de su formación.

La linealidad de la distribución de los máseres en las regiones de formación de estrellas de gran masa es un fenómeno que se repite frecuentemente. Desde que se comenzaron a realizar los primeros relevamientos de máseres se encontró que estos yacen en grupos que tienden a formar distribuciones lineales (o elongadas). En las regiones analizadas en este estudio se encontró que la linealidad es un fenómeno muy común en la distribución de los máseres ya que 7 de las 8 regiones estudiadas evidencian distribuciones lineales 0 bien elongadas.

Los alineamientos geométricos de los máseres con estructura de discos y/o "jets" han sido ampliamente utilizados en la literatura. Sin embargo presentan algunas ventajas y limitaciones. Entre las ventajas podemos mencionar que es una técnica muy expeditiva ya que sólo se requiere de las posiciones "offsets" de los máseres individuales. Entre las limitaciones se encuentra el hecho de que sin información adicional no es posible descartar la posibilidad de alineamientos casuales. La elevada frecuencia de los mismos en regiones de formación de estrellas de gran masa minimiza la probabilidad de que un número significativo de ellos sean alineamientos sólo al azar, sin embargo no permite asegurar que una configuración, en particular, no pueda deberse a factores ajenos a las estructuras de discos y/o "jets". Además los alineamientos por si mismos tampoco permiten determinar la cinemática de la estructura ya sea disco o "jet/outflow" con la que están asociados. 
En algunos casos los alineamientos no son tan evidentes o resulta difícil asociar los máseres con las estructuras de discos y/o "jets" solamente en base a la geometría de los mismos. Además las emisiones de máseres son variables en diversas escalas temporales lo cual puede dificultar la identificación de los alineamientos. En estos casos resulta de gran utilidad el empleo de los movimientos propios de los máseres en cuestión.

La determinación de los movimientos propios de los máseres puede proporcionar información sobre la cinemática de las estructuras que ellos trazan, además de permitir asegurar el significado físico de los alineamientos detectados y descartar efectos casuales. La desventaja principal de esta técnica es que por el momento no es muy expeditiva ya que se requiere del uso de grandes radiotelescopios como el "Very Long Baseline Interferometry (VLBI)", motivo por el cual, en la actualidad, ha sido aplicada sólo a un número relativamente muy pequeño de regiones.

Para determinar movimientos propios se requiere alta resolución angular y períodos relativamente largos (algunos meses). Hacemos notar que aun así los movimientos propios obtenidos son muy pequeños. Para fijar ideas un máser que yace a una distancia de $0.5 \mathrm{Kpc}$ (la mayoría de las regiones de formación de estrellas de masa alta yacen a varios $\mathrm{Kpc}$ ) y se mueven a una velocidad de $10 \mathrm{Kms}^{-1}$ tendría un movimiento propio de 0.4 milisegundos de arco por mes. Movimientos propios tan pequeños son detectables sólo, como se dijo, con grandes radiotelescopios de altísima resolución angular.

En cuanto al rol de los máseres en la formación de las estrellas de gran masa, éste parece estar principalmente relacionado con la posibilidad que brindan de detectar y estudiar las estructuras de discos y "jets" en regiones lejanas altamente oscurecidas donde se forman estrellas de gran masa. Sin embargo no parecerían estar vinculados al proceso de formación estelar en sí. No lo desencadena ni lo inhiben. Tampoco parecerían influir en el proceso de formación mismo. Es decir que las emisiones de máseres permiten revelar el nacimiento de las nuevas estrellas de gran masa y sin modificarlo o condicionarlo de alguna manera. Los máseres existen asociados con discos y/o "jets" en estrellas de gran masa porque en ellas se dan las condiciones físicas para su excitación y no porque sean necesarios para la formación de estrellas de gran masa mismas.

\section{Conclusiones}

En este trabajo hemos realizado un estudio global sobre la distribución de máseres de metanol, hidroxilo y agua en 8 regiones de formación de estrellas de gran masa. Buscamos alineamientos que pudieran dar indicios de posibles vínculos físicos entre los máseres y las estructuras de discos y/o "jets/outflows" para cada una de las regiones estudiadas. A continuación sintetizamos nuestras conclusiones:

- Las emisiones de máseres que circundan las protoestrellas de masa alta tienden a formar grupos que en un porcentaje muy elevado muestran distribuciones lineales o elongadas.

- El comportamiento de los máseres en las regiones de formación de estrellas de gran masa es efectivamente dicotómico. Pueden trazar tanto estructuras de discos como de "jets".

- Encontramos que no existe una marcada preferencia de los máseres de metanol, hidroxilo y agua a alinearse con discos y/o "jets/outflows". En general, los máseres tienden a alinearse con los discos y los "jets" en proporciones similares. Sólo advertimos una ligera tendencia de los máseres de agua a trazar 0 (delinear) "outflows".

- El hecho de que los máseres puedan trazar tanto discos como "jets" presenta un aspecto positivo de gran importancia para el estudio del proceso de formación de las estrellas de gran masa el cual se produce en regiones altamente oscurecidas en el óptico e incluso en el infrarrojo cercano y medio. La emisión de máser puede "escapar" de la nube madre y nos permite "ver" los discos y "jets" en estrellas de gran masa que de otra manera son muy difíciles de detectar. 
- Nuestro estudio se basa en la identificación de alineamientos geométricos de máseres de distintos tipos en las proximidades de las estrellas de gran masa. La elevada frecuencia de los mismos apoya la hipótesis de que no se trata simplemente de alineamientos casuales. Sin embargo la determinación de los movimientos propios de los mismos puede eliminar cualquier tipo de ambigüedad y aportar información cinemática de los mismos que permitan establecer, fehacientemente, la cinemática de los discos y/o "jets".

- Los máseres existen asociados con los discos y/o "jets" en estrellas de gran masa porque en ellos se dan las condiciones físicas para su excitación y no porque sean necesarios para la formación de las estrellas de gran masa misma. Es decir que no son los desencadenantes del proceso de colapso que las origina, tampoco lo aceleran o lo inhiben.

\section{Bibliografía}

1. Goddi, $\mathrm{C}_{\text {.; }}$ et al. "Kinematics of $\mathrm{H}_{2} \mathrm{O}$ masers in high-mass star forming regions". Astronomy and Astrophysics. 2005: (432): 161-173.

2. Qin, S-L.; et al. "Massive molecular outflows associated with UCHII/HII regions". Astronomy and Astrophysics. 2008: (484): 361-369.

3. Tej, A.; et al. "A multiwavelength study of the massive star-forming region IRAS 06055+2039 (RAFGL 5179)". Astronomy and Astrophysics. 2006: (452): 203-215.

4. Umanzor, J. y Gómez, M.: "Emisiones Máseres en Regiones de Estrellas de Gran masa: ¿Trazadores de Discos o de Jets?" (Tesis de Maestría). Tegucigalpa: Observatorio Astronómico Centroamericano de Suyapa de la Universidad Nacional Autónoma de Honduras. 2008.

5. Xu, Y.; et al. "Molecular Outflows around High-Mass Young Stellar Objects". The Astronomical Journal. 2006. (132): 20-26. 